\title{
Otimização de método analítico em HPLC-PDA para monitoração terapêutica do bussulfano oral em pacientes transplantados de células-tronco hematopoiéticas
}

\author{
Optimization of an HPLC-PDA analytical method for therapeutic monitoring of oral busulfan in \\ hematopoietic stem cell transplant patients \\ Optimización de un método analítico HPLC-PDA para la monitorización terapéutica de busulfán oral en \\ pacientes con trasplante de células madre hematopoyéticas
}

Recebido: 07/05/2021 | Revisado: 18/05/2021 | Aceito: 21/05/2021 | Publicado: 07/06/2021

Cristiane Effting

ORCID: https://orcid.org/0000-0002-6544-7271 Universidade Estadual de Goiás, Brasil

E-mail: cristiane.effting@ueg.br

Fernando Gomes Ferreira Oliveira

ORCID: https://orcid.org/0000-0001-9691-6440

New York Pain Refief Medicine, Estados Unidos E-mail: fernandogfoliveira@gmail.com

Iram Moreira Mundim

ORCID: https://orcid.org/0000-0002-1720-1840

Instituto de Ciências Farmacêuticas, Brasil

E-mail: iram.mundim@icf.com.br

Alessandro de Carvalho Cruz

ORCID: https://orcid.org/0000-0002-8878-1678

Universidade Federal de Goiás, Brasil

E-mail: alesscruz@yahoo.com.br

Andryne Rego Rodrigues

ORCID: https://orcid.org/0000-0001-5129-9362

Universidade Federal de Goiás, Brasil

E-mail: andrynerodrigues@hotmail.com

Caroline Rego Rodrigues

ORCID: https://orcid.org/0000-0002-2560-6725

Universidade Estadual de Goiás, Brasil

E-mail: rodrigues.caroline.r@gmail.com

Jerônimo Raimundo de Oliveira Neto

ORCID: https://orcid.org/0000-0002-0261-4554

Universidade Federal de Goiás, Brasil

E-mail: jeronimoneto8@gmail.com

Marianna Medeiros Barros da Cunha

ORCID: https://orcid.org/0000-0003-1655-6938

Universidade Federal de Goiás, Brasil

E-mail: mariannammbc@gmail.com

Adriano de Moraes Arantes

ORCID: https://orcid.org/0000-0002-3866-3083

Hospital Araújo Jorge da Associação de Combate ao Câncer de Goiás, Brasil

E-mail: arantesadriano@ hotmail.com

Luiz Carlos da Cunha

ORCID: http://orcid.org/0000-0002-1525-8528

Universidade Federal de Goiás, Brasil

E-mail: lucacunha@gmail.com

\begin{abstract}
Resumo
Otimizou-se método para monitoração plasmática de bussulfano em pacientes submetidos a transplante de célulastronco hematopoiéticas (TCTH). Utilizou-se HPLC-PDA Shimadzu, coluna C18 (150 mm x $4 \mathrm{~mm})$, metanol/água/acetonitrila $(65: 20: 15, \mathrm{v} / \mathrm{v} / \mathrm{v})$, fluxo $1 \mathrm{~mL} \min -1$; UV $\lambda=276 \mathrm{~nm}$, tempo de análise de $17 \mathrm{~min}$; derivatização com dietilcarbamato de sódio, extração com acetato de etila; curva de calibração linear de 200-5000 ng mL-1. A recuperação relativa dos controles foi de 89\% $\pm 0,04(\mathrm{CQA}=86 \%$; CQM = 87\%; CQB = 93\%) e a do PI (padrão interno) foi de 74\% $\pm 0,47$. A recuperação absoluta dos controles foi de $114 \% \pm 0,03(\mathrm{CQA}=111 \%$; CQM = $118 \%$; CQB $=113 \%$ ), e a do PI foi de $102 \% \pm 2,5$. Os limites inferiores de quantificação e detecção obtidos foram, respectivamente, de $200 \mathrm{ng} \mathrm{mL}^{-1}$ e $40 \mathrm{ng} \mathrm{mL}^{-1}$. A linearidade foi analisada pela curva de calibração (200-5000 ng. $\left.\mathrm{mL}^{-1}\right)(\mathrm{r}=0,998)$. A repetibilidade (intracorrida) foi de $1,25 \%-11,25 \%$, e a precisão intermediária (intercorrida), 2,17\%-10,71\%. A exatidão encontrada foi de $89,61 \%-102,18 \%$. A estabilidade de curta duração de amostras
\end{abstract}


extraídas e das soluções foi de $6,25 \%$ e $3,18 \%$ para CQB, $7,54 \%$ e 2,4\% para CQA e $13,17 \%$ e $4,13 \%$ para PI, respectivamente. A estabilidade de ciclos de congelamento e descongelamento de amostras extraídas e das soluções foi de $3,64 \%$ e $2,53 \%$ para CQB, $9,09 \%$ e $5,65 \%$ para CQA e $10,32 \%$ e $4,82 \%$ para PI, respectivamente. Desta forma, foi possível validar uma técnica para determinação do bussulfano em plasma por HPLC-PDA, adequada à monitorização terapêutica de pacientes.

Palavras-chave: Busulfano; HPLC; Monitoramento de medicamentos.

\begin{abstract}
The study aims to develop activities for therapeutic monitoring of oral busulfan in patients undergoing heterologous stem-cell transplantation (HSCT) through the identification and determination of plasma busulfan. It was used HPLC-PDA Shimadzu C18 column (150 mm x $4 \mathrm{~mm})$, methanol/water/acetonitrile (65:20:15, v/v/v), flow rate 1 $\mathrm{mL}$ min-1, UV $\lambda=276 \mathrm{~nm}$, analysis time $17 \mathrm{~min}$; derivatization with sodium diethylcarbamate, extraction with ethyl acetate; linear calibration curve over the range of 200-5000 ng ml-1. The average relative recovery of the controls was $89 \% \pm 0.04(\mathrm{QCH}=86 \%$; QCM=87\%; QCL=93\%), and the internal standard (IS) was 74\% \pm 0.47 . The mean absolute recovery of the controls was $114 \pm 0.03 \%(\mathrm{QCH}=111 \%, \mathrm{QCM}=118 \% ; \mathrm{QCL}=113 \%)$, and the IS was $102 \pm 2.5 \%$. The lower limits of detection and quantification obtained were respectively $200 \mathrm{ng} \mathrm{ml}-1 \mathrm{and} 40 \mathrm{ng} . \mathrm{mL}-$ 1. The linearity was evaluated by calibration curve (200-5000 ng.mL-1) ( $r=0.998, r=0.994, r=0.998)$. The repeatability (intracorrida) resulted in $1.25 \%-11.25 \%$ and intermediate precision (intercorrida) resulted in $2.17 \%$ $10.71 \%$. The accuracy was found to be $89.61 \%-102.18 \%$. The short-term stability of the solutions and samples extracted was $6.25 \%$ and $3.18 \%$ for QCL, $7.54 \%$ and $2.4 \%$ for QCH, and $4.13 \%$ and $13.17 \%$ for IS, respectively. The stability of freeze-thaw cycles extracted samples and the solutions was $3.64 \%$ and $2.53 \%$ for QCL, 9.09\% and $5.65 \%$ and QCH to $4.82 \%$ and $10.32 \%$ for IS, respectively. It was concludefd that it was possible to describe a technique for determining the busulfan in plasma by HPLC-PDA, settling their validation parameters for therapeutic drug monitoring purposes.
\end{abstract}

Keywords: Busulfan; HPLC; Therapeutic drug monitoring.

\title{
Resumen
}

Se optimizó un método para la monitorización plasmática de busulfán en pacientes sometidos a trasplante de células madre hematopoyéticas (TCMH). HPLC-PDA Shimadzu, columna C18 (150 mm x $4 \mathrm{~mm})$, metanol/agua/acetonitrilo (65:20:15, v/v/v), se usó un flujo de $1 \mathrm{ml} \mathrm{min-1;} \mathrm{UV} \lambda=276 \mathrm{~nm}$, tiempo de análisis 17 min; derivatización con dietilcarbamato de sodio, extracción con acetato de etilo; curva de calibración lineal de 200-5000 ng.mL-1. La recuperación relativa de los controles fue de 89\% $\pm 0,04$ (CQA = 86\%; CQM = 87\%; CQB $=93 \%$ ) y la del EI (estándar interno) fue de $74 \% \pm 0,47$. La recuperación absoluta de los controles fue $114 \% \pm 0.03$ $(\mathrm{CQA}=111 \% ; \mathrm{CQM}=118 \% ; \mathrm{CQB}=113 \%)$, y la del EI fue 102\% \pm 2.5 . Los límites inferiores de cuantificación y detección obtenidos fueron, respectivamente, 200 ng.mL-1 y 40 ng.mL-1. La linealidad se analizó mediante la curva de calibración (200-5000 ng.mL-1) ( $\mathrm{r}=0$,998). La repetibilidad (intra-análisis) fue de 1,25\% $-11,25 \%$ y la precisión intermedia (entre análisis), 2,17\% -10,71\%. La precisión encontrada fue de 89,61\% $-102,18 \%$. La estabilidad a corto plazo de las muestras y soluciones extraídas fue de $6.25 \%$ y $3.18 \%$ para CQB, $7.54 \%$ y $2.4 \%$ para CQA y $13.17 \%$ y $4.13 \%$ para EI, respectivamente. La estabilidad de los ciclos de congelación y descongelación de las muestras y soluciones extraídas fue de $3.64 \%$ y $2.53 \%$ para CQB, 9.09\% y 5.65\% para CQA y $10.32 \%$ y $4.82 \%$ para EI, respectivamente. Así, fue posible validar una técnica para la determinación de busulfán en plasma por HPLC-PDA, adecuada para monitorización terapéutica de los pacientes.

Palabras clave: Busulfán; HPLC; Monitorización terapéutica.

\section{Introdução}

O bussulfano, ou 1,4-dimetanosulfoniloxibutano, é um éster derivado do ácido sulfônico e agente alquilante utilizado na prática clínica desde 1950, com potente atividade antitumoral e intensa propriedade de suprimir a proliferação de células mieloides (Ciurea \& Anderson, 2009).

Embora o bussulfano tenha sido bastante utilizado como tratamento paliativo da leucemia mieloide crônica e de leucemias granulocíticas, com o advento do mesilato de imatinibe, suas utilizações clínicas ficaram bastante restritas. Atualmente, porém, ainda apresenta importante aplicação para o regime de condicionamento em pacientes submetidos a transplante de células-tronco hematopoiéticas (TCTH). Para essa finalidade, é utilizado em altas doses (1 mg kg-1 a cada $6 \mathrm{~h}$ por 16 doses), em associação com ciclofosfamida, nos chamados esquemas de condicionamento antes da infusão de célulastronco (Chow et al., 1997; Bleyzac et al., 2000; Bullock et al., 2006; Ciurea \& Anderson, 2009). As funções do bussulfano e 
da ciclofosfamida são complementares, com o primeiro realizando a ablação da medula e a segunda destruindo os linfócitos maduros, suprimindo o organismo receptor e habilitando-o a receber o transplante (Slattery \& Risler, 1998).

A associação bussulfano/ciclofosfamida tem sido utilizada como alternativa à irradiação corporal total (ICT), pois possui eficácia semelhante à ICT, é mais bem tolerada e tem sido associada à diminuição da toxicidade relacionada ao tratamento, maior sobrevida, menor possibilidade de recaídas e, possivelmente, menor risco de desenvolver a doença do enxerto contra o hospedeiro (DECH) (Chow et al., 1997; Slattery et al., 1997; Takamatsu et al., 2000; Bornhäuser et al., 2003; Bullock et al., 2006).

A monitoração das concentrações plasmáticas no estado de equilíbrio $\left(\bar{C}_{s s}\right)$ e da área sob a curva (ASC) versus tempo do bussulfano tem sido utilizada para testar a associação com a incidência de toxicidade hepática e de mortalidade relacionada ao transplante, além da recaída da doença primária e da rejeição do enxerto (Tran et al., 2000).

Pacientes que recebem bussulfano oral apresentam ampla variação nas taxas de absorção e de biodisponibilidade (Slattery et al., 1997; Tabak et al.,2001). Já foi demonstrado que a área sob a curva total $\left(\mathrm{ASC}_{\mathrm{T}}\right)$ e a $\overline{C_{s S}}$ variam extensamente (até 25 vezes) entre pacientes após uma dose oral e que esta variação pode ter grande impacto no desfecho do transplante (Takamatsu et al., 2005). A extrema variação na biodisponibilidade e na farmacocinética do bussulfano oral levaram à descoberta da relação entre farmacocinética e desfechos clínicos, como rejeição de enxertia e toxicidade (Ciurea \& Anderson, 2009).

A doença veno-oclusiva hepática (DVOH) ou síndrome de obstrução sinusoidal (SOS) é a mais séria manifestação de toxicidade dose-limitante ao tratamento com bussulfano, tanto no transplante de pacientes adultos, como pediátricos, embora a mucosite seja a mais comum. É uma síndrome clínica caracterizada por retenção de fluidos, dor, hepatomegalia, icterícia, ascite e/ou ganho de peso inexplicáveis podendo levar à falência de múltiplos órgãos e morte (Slattery \& Risler, 1998). Valores de $\bar{C}_{s S}$ maiores do que $900 \mathrm{ng} \mathrm{mL}^{-1}$ aumentam o risco de SOS, enquanto concentrações menores do que $600 \mathrm{ng} \mathrm{mL}^{-1}$ aumentam o risco de rejeição do enxerto. Baixos valores de $\mathrm{ASC}_{\mathrm{T}}$ também estão associados a maiores riscos de recaídas em pacientes com leucemia mieloide crônica. A individualização da dose e a monitoração terapêutica podem ser utilizadas como estratégias clínicas para manter a $\bar{C}_{s S}$ do bussulfano entre $600 \mathrm{ng} \mathrm{mL}^{-1}$ e $900 \mathrm{ng} \mathrm{mL}^{-1}$ e melhorar o desfecho dos transplantes (Tabak et al., 2001; Bullock et al., 2006; Nath \& Shaw, 2007; Corbacioglu, Jabbour \&, Mohty, 2019; Philippe et al., 2019; Feng et al., 2020).

Salman e colaboradores (2019) monitoraram e ajustaram a dose do bussulfano para 800-900 ng/ml e observaram 17\% de DVOH. Segundo os autores, o ajuste da dose pode ter contribuído para o resultado, embora mais estudos sejam necessários para excluir outros fatores, com por exemplo, genéticos. Philippe et al., 2019 relatam a concentração máxima de bussulfano como um forte preditor de DVOH, sendo descrito como uma reação dependente da concentração (Salman et al., 2019; Philippe et al., 2019).

Lawson e colaboradores realizaram revisão da literatura sobre a farmacocinética do bussulfano em pacientes pediátricos e encontraram relação entre maiores concentrações terapêuticas do fármaco e a DVOH. Afirmam, ainda, a necessidade de monitoramento da exposição ao bussulfano durante todo o condicionamento e a necessidade de mais estudos sobre os efeitos dos metabólitos do bussulfano e de outros fatores possivelmente associados a DVOH (Lawson, Staatz \&, Hennig, 2021).

Outras manifestações de reações adversas que podem ocorrer envolvem mucosite grave, irritação gastrintestinal, neurotoxicidade, cardiotoxicidade, cistite hemorrágica e pneumonia intersticial (Chow et al., 1997; Hassan et al., 2000). 
Visando desenvolver atividades de monitoração terapêutica de bussulfano oral em pacientes com doenças mieloproliferativas e síndromes mielodisplásicas submetidos a transplante alogênico mieloablativo no estado de Goiás (Effting et al., 2015), desenvolveu-se uma técnica analítica em cromatografia líquida de alta eficiência acoplada a detector de arranjo de diodos (HPLC-PDA), que foi validada para a identificação e a dosagem plasmática do bussulfano, para posterior aplicação a pacientes. A disponibilidade do método pode ajudar a diminuir a toxicidade do bussulfano em pacientes que estejam em tratamento com o medicamento.

\section{Material e Métodos}

\subsection{Parte Experimental}

O protocolo para este estudo, incluindo a utilização de amostras biológicas com a finalidade de quantificação do bussulfano oral, foi aprovado pelo Comitê de Ética em Pesquisa (CEP) do Hospital Araújo Jorge da Associação de Combate ao Câncer em Goiás (ACCG) (Protocolo CEPACCG nº 007/09).

\section{Reagentes e materiais}

Acetato de etila, acetonitrila e metanol grau HPLC (Tedia Brazil, Rio de Janeiro, RJ, Brasil); dietilditiocarbamato de sódio (Sigma-Aldrich, Steinheim, Alemanha); bussulfano (Fluka Analytical, Sigma-Aldrich, Steinheim, Alemanha); 1,6bis(metanesulfoniloxy)hexano (padrão interno - PI), sintetizado e doado pela aluna de doutorado Francine Attié de Castro, sob a orientação da Profa. Dra. Vera Lucia Lanchote, do Laboratório de Farmacocinética e Metabolismo da Faculdade de Ciências Farmacêuticas de Ribeirão Preto, Universidade de São Paulo; água ultrapura, obtida diariamente utilizando-se o sistema MilliQ (Millipore Corporation, Chicago, IL, Estados Unidos).

Os seguintes fármacos foram gentilmente cedidos para a avaliação do ensaio de especificidade: aciclovir, albendazol, dipirona, fluconazol, omeprazol, sulfametoxazol + trimetropina e vancomicina (Genix, Anápolis, GO, Brasil), anfotericina B, ondansetrona (Cristália, Itapira, SP, Brasil), cefalotina, meropenem (Cellofarm, Rio de Janeiro, RJ, Brasil), dimenidrinato (Nycomed, Jaguariúna, SP, Brasil), cefepime (Biochimico, Rio de Janeiro, RJ, Brasil) e tramadol (Hipolabor, Sabará, MG, Brasil).

\section{Instrumentação e condições cromatográficas de análise}

As análises quantitativas de bussulfano em plasma humano foram realizadas por meio de cromatógrafo líquido de alta eficiência LC-20AT (Shimadzu, Quioto, Japão), equipado com DAD (SPD-M20A) e detector de fluorescência (RF-10AXL), acoplado a um amostrador automático SIL-20A (Prominence Autosampler) e forno de colunas CTO-20A (Prominence Column Oven), controlados por módulo de comunicação CBM-20A.

As análises foram realizadas com coluna $\mathrm{ACE}^{\circledR} 5 \mathrm{C} 18(150 \mathrm{~mm}$ x $4 \mathrm{~mm})$, tamanho da partícula $5 \mu \mathrm{m}$, acoplada à précoluna $\mathrm{ACE}^{\circledR} 5 \mathrm{C} 18$, tamanho da partícula $5 \mu \mathrm{m}$. A fase móvel utilizada foi metanol:água:acetonitrila (65:20:15, v/v/v), degaseificada com o auxílio de banho de ultrassom antes de ser colocada no sistema, sob fluxo de $1 \mathrm{~mL} \mathrm{~min}{ }^{-1}$, padrão interno 1,6-bis-(metanosulfoniloxi) hexano, utilizando detector DAD e $\lambda=276 \mathrm{~nm}$.

As corridas analíticas foram monitoradas em registrador e integrador Shimadzu acoplados a microcomputador com software LC Solution para registro, integração, gerenciamento e tratamento dos dados analíticos.

\section{Equipamentos}

Balança analítica de precisão (Celtac, Curitiba, PR, Brasil), agitador de tubos tipo vortex AP 56 (Phoenix, Curitiba, PR, Brasil), concentrador de amostras TE-019 (Tecnal, Piracicaba, SP, Brasil), lavadora ultrassônica UltraClear (Unique, 
Indaiatuba, SP, Brasil), centrífuga Excelsa (Fanem, Guarulhos, SP, Brasil) e microcentrífuga CM-610 (Biosystems, Curitiba, PR, Brasil).

\section{Preparação de solução padrão e reagentes}

O bussulfano $(10 \mathrm{mg}$ ) foi pesado e, posteriormente, dissolvido e homogeneizado em acetato de etila grau HPLC em balão volumétrico de $100 \mathrm{~mL}$. Foram feitas diluições seriadas de 50000, 40000, 32000, 21500, 16000, 8000, 4000, 3000 e $2000 \mathrm{ng} \mathrm{mL}^{-1}$, para uso como soluções de trabalho de bussulfano - padrão (STD). De modo semelhante, uma solução de 1,6bis(metanossulfoniloxi)hexano $\left(25 \mu \mathrm{g} \mathrm{mL}^{-1}\right)$ foi preparada em acetato de etila grau HPLC para uso como PI, sendo tais soluções utilizadas para diluição em plasma. Todas as soluções (STD e PI) assim obtidas foram estocadas à temperatura de $20^{\circ} \mathrm{C}$. A solução de derivatizante foi preparada diariamente dissolvendo-se $0,82 \mathrm{~g}$ de dietilditiocarbamato de sódio (DDTC) em 10 mL de água ultrapura (Heggie et al., 1997).

Derivatização e extração do plasma

Durante a validação da metodologia analítica, foram utilizadas bolsas de plasma doadas pelo Banco de Sangue do Hospital das Clínicas da Universidade Federal de Goiás (UFG) e do Instituto Goiano de Oncologia e Hematologia (INGOH). Ressaltamos que o método foi desenvolvido a partir de diversos testes e tentativas de adequação às nossas condições laboratoriais, baseando-se em, pelo menos, quatro artigos: Chow et al., 1997; Heggie et al., 1997; Bleyzac et al., 2000; Tran et al., 2000.

As amostras da curva de calibração (200, 400, 800, 1600, 3200 e $5000 \mathrm{ng} \mathrm{mL}^{-1}$ ) foram injetadas em duplicata

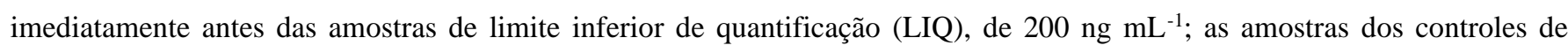
qualidade baixo (CQB), médio (CQM) e alto (CQA), de $300 \mathrm{ng} \mathrm{mL}{ }^{-1}, 2150 \mathrm{ng} \mathrm{mL}^{-1}$ e $4000 \mathrm{ng} \mathrm{mL}^{-1}$, respectivamente, foram preparadas em sextuplicata em tubos tipo Eppendorf de $2 \mathrm{~mL}$. Foram adicionados $50 \mu \mathrm{L}$ das STD e PI ao plasma (500 $\mu \mathrm{L}$ ), acrescentando-se $100 \mu \mathrm{L}$ da solução de DDTC e submetendo-se a agitação no vórtex por 10 s; em seguida, foi feita adição de $500 \mu \mathrm{L}$ de acetonitrila, submetendo-se a mais 60 s de agitação em vórtex e 5 min de centrifugação a 6000 rpm. O sobrenadante foi transferido para tubos de vidro limpos $(12 \mathrm{~cm} \mathrm{x} 1,1 \mathrm{~cm})$, sendo, em seguida, adicionados $2 \mathrm{~mL}$ de acetato de etila e submetendo-se a agitação por 60 s no vórtex. As amostras foram centrifugadas por 10 min a $3500 \mathrm{rpm}$; a fase orgânica (2 mL) foi transferida para tubos de vidro limpos $(7,5 \mathrm{~cm}$ x $0,8 \mathrm{~cm})$, os quais foram colocados para evaporação até secura sob fluxo de oxigênio a $50^{\circ} \mathrm{C}$. A amostra seca foi dissolvida com $100 \mu \mathrm{L}$ da fase móvel, submetida à agitação por $60 \mathrm{~s}$ no vórtex e transferida para tubos (vials) em estante e acondicionados no amostrador automático do HPLC para injeção de $30 \mu \mathrm{L}$ de cada amostra.

As curvas de calibração no plasma consistiram em branco (com derivatizante), branco (com PI), mais as seis concentrações citadas, em duplicata, e os controles em sextuplicata, por três dias, para avaliação de linearidade, precisão e exatidão.

\section{Procedimentos de validação}

A adequação do sistema foi avaliada durante a corrida cromatográfica de acordo com os parâmetros do Internacional Conference on Harmonization ( $\mathrm{ICH}, 2005)$. Os parâmetros cromatográficos observados foram o número de pratos teóricos (N), o fator de capacidade $\left(\mathrm{k}^{\prime}\right)$, a simetria do pico e a resolução entre os picos.

Para a validação do método, foram utilizados como parâmetros os testes recomendados na Resolução - RE $n^{\circ} 899$, da Agência Nacional de Vigilância Sanitária (ANVISA) do Brasil, os quais são descritos a seguir (Brasil, 2003).

\section{a) Especificidade}


Em métodos cromatográficos com auxílio de detector DAD, pode-se avaliar a pureza do pico cromatográfico por meio da verificação de possíveis interferentes à substância de interesse no mesmo tempo de retenção do analito em estudo. Dessa forma, a especificidade foi testada contra os fármacos aciclovir, albendazol, anfotericina B, cefalotina, cefepime, dimenidrinato, dipirona, fluconazol, meropenem, omeprazol, ondansetrona, sulfametoxazol + trimetropina, tramadol e vancomicina, dentro de concentrações terapêuticas, nas mesmas condições utilizadas para a análise do bussulfano, com corrida de 30 min, para verificação de possível interferência no tempo de retenção e comprimento de onda do estudo. Estes testes foram realizados com amostras de plasma livre de bussulfano de seis indivíduos, sendo quatro normais, uma amostra lipêmica e uma hemolisada.

\section{b) Linearidade}

A linearidade foi determinada pela construção da curva de calibração que demonstra a relação entre a resposta do detector e a concentração conhecida do analito. Foram realizadas duplicatas diárias de seis concentrações de bussulfano (200,

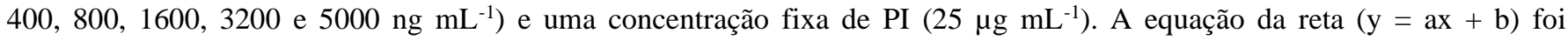
determinada calculando-se o intercepto (b), a inclinação (a) e o coeficiente de correlação linear (r). A linearidade foi estimada por análise de regressão linear pelo método dos mínimos quadrados.

\section{c) Limites de detecção e de quantificação}

A estimativa do limite de detecção foi realizada com base na razão sinal-ruído equivalente a três vezes o ruído da linha de base. O limite de quantificação também foi determinado desta forma, porém na razão superior a 10:1.

\section{d) Precisão e exatidão}

A precisão (intra e inter-ensaio) do método foi determinada em três dias diferentes, com sextuplicatas diárias de amostras com concentrações de LIQ, CQB, CQM e CQA. A precisão do método analítico foi expressa como a estimativa do desvio padrão relativo (DPR).

A exatidão foi realizada por ensaios em triplicatas diárias de amostras com as concentrações citadas acima. Os resultados obtidos devem ser diretamente proporcionais à concentração do analito na amostra, dentro do intervalo de $80 \%$ a $120 \%$ da concentração teórica do teste, de acordo com a Equação 1:

$$
\text { Exatidão }(\%)=\left(\frac{\mathrm{CME}}{\mathrm{CT}}\right) \times 100 \quad \text { Equação } 1
$$

em que CME é a concentração média experimental e CT é a concentração teórica.

\section{e) Estabilidade}

Para verificação da estabilidade de curta duração em amostras extraídas, analisaram-se três amostras nas concentrações de CQB e CQA armazenadas por $6 \mathrm{~h}$ e por $10 \mathrm{~h}$ a temperatura ambiente (TA). A estabilidade ao congelamento e descongelamento foi verificada após a submissão das soluções e amostras extraídas (nas concentrações de CQB e CQA) a três ciclos de congelamento a $-20^{\circ} \mathrm{C}(24 \mathrm{~h})$ seguido de descongelamento a TA. Os resultados foram comparados com os obtidos a partir de amostras recém-preparadas. 


\section{f) Recuperação}

Injetaram-se oito amostras extraídas e oito não extraídas (preparadas em fase móvel) nas concentrações de CQB, CQM e CQA, obtendo-se as áreas dos picos de bussulfano e PI. O resultado foi expresso em percentagem da quantidade obtida da comparação dos resultados analíticos de soluções padrão não extraídas, conforme a Equação 2:

$$
\text { Recuperação }(\%)=\frac{\text { Média áreas extraídas }}{\text { Média áreas não extraídas }} \times 100 \text { Equação } 2
$$

O teste de recuperação absoluta ou efeito matriz foi realizado para observar o efeito do plasma (matriz) sobre o processo de extração. Para tanto, foram preparadas amostras brancas, a partir do processo de precipitação do plasma com acetonitrila e extração com acetato de etila com a transferência da fase orgânica para outro tubo seguida de secagem. Posteriormente, foram adicionados o PI, o STD e o derivatizante e a amostra foi novamente seca, reconstituída com a fase móvel e injetada. As áreas dos picos assim obtidas foram comparadas com as áreas dos picos obtidas de soluções em fase móvel (não extraídas), conforme a Equação 2.

\section{Resultados}

Na Tabela 1, são mostrados os valores obtidos durante o desenvolvimento da técnica analítica, bem como os valores de referência encontrados na literatura para os parâmetros cromatográficos. Os resultados obtidos encontraram-se dentro dos valores de referência requeridos (ICH, 2005).

Tabela 1. Condições cromatográficas do método analítico de análise do bussulfano oral em amostras de plasma.

\begin{tabular}{|c|c|c|c|}
\hline Parâmetro & Bussulfano & PI & $\mathrm{ICH}$ \\
\hline Fator de capacidade $\left(\mathrm{k}^{\prime}\right)$ & 5,1 & 8,5 & $>2,0$ \\
\hline Número de pratos teóricos & 4659 & 4986 & $>2000$ \\
\hline Simetria do pico & 1,5 & 1,2 & $\leq 2,0$ \\
\hline Resolução entre os picos & \multicolumn{2}{|c|}{4,0} & $>2,0$ \\
\hline
\end{tabular}

PI = padrão interno; $\mathrm{ICH}=$ recomendado pelo ICH (2005). Fonte: Autores.

Na Figura 1 pode-se comparar o cromatograma branco a um cromatograma com os picos de interesse e ainda dois cromatogramas sobrepostos ao branco que demonstram a ausência de interferentes nos tempos de retenção dos picos. Da mesma forma, as amostras de plasma lipêmico e hemolisado não interferiram na técnica analítica. Os fármacos testados aciclovir, albendazol, anfotericina B, cefalotina, cefepime, dimenidrinato, dipirona, fluconazol, meropenem, omeprazol, ondansetrona, sulfametoxazol + trimetropina, tramadol e vancomicina - não foram detectados nos tempos de retenção e nos comprimentos de onda de interesse, o que demonstra a especificidade da técnica para os propósitos de controle terapêutico. 
Figura 1 - Cromatogramas representativos da validação analítica do bussulfano em amostras de plasma, por HPLC-PDA.

(A)

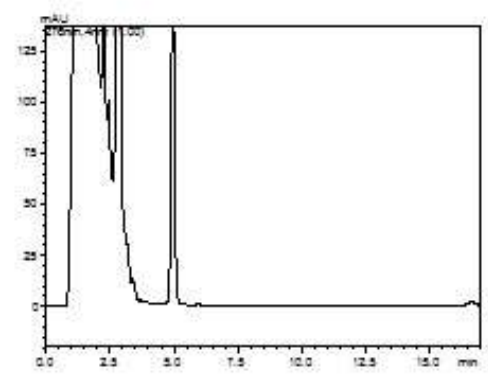

(C)

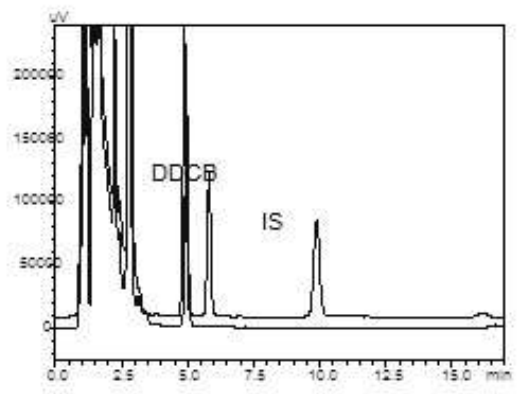

(B)

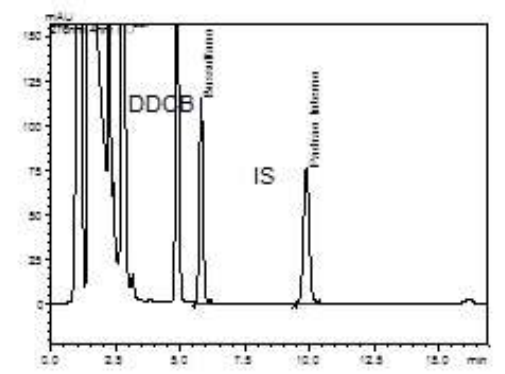

(D)

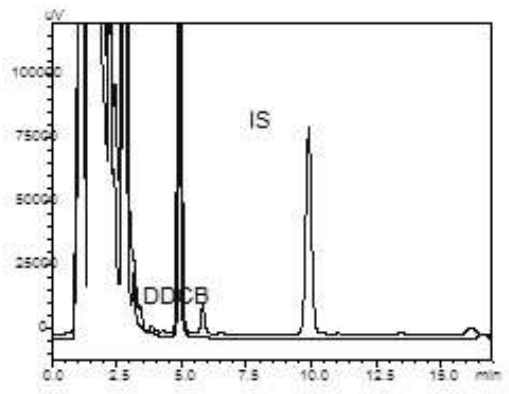

(A) Cromatograma branco. (B) Cromatograma do bussulfano derivatizado [(1,4- bis(dietilditiocarbamoil) butano] (DDCB) e padrão interno derivatizado (PI) em amostra extraída com acetonitrila e acetato de etila na concentração $2150 \mathrm{ng} \mathrm{mL}^{-1}$. (C) Cromatograma branco sobreposto ao cromatograma do DDCB e PI em amostra extraída com acetonitrila e acetato de etila na concentração de $2150 \mathrm{ng} \mathrm{mL}^{-1}$. (D) Cromatograma branco sobreposto ao cromatograma do DDCB e PI em amostra extraída com acetonitrila e acetato de etila na concentração de $200 \mathrm{ng} \mathrm{mL} \mathrm{m}^{-1}$. Fonte: Autores.

As curvas de calibração obtidas demonstraram ser lineares na faixa de concentração testada $\left(200-5000 \mathrm{ng} \mathrm{mL}^{-1}\right)(\mathrm{r}=$ 0,998; $r=0,994 ; r=0,998)$. Os limites inferiores de quantificação e deteç̧ão obtidos neste estudo foram, respectivamente, de $200 \mathrm{ng} \mathrm{mL}^{-1}$ e $40 \mathrm{ng} \mathrm{mL}^{-1}$.

Os valores médios de concentrações de bussulfano em amostras de plasma obtidos no limite inferior de quantificação, controles de qualidade baixo, médio e alto durante a validação do método analítico, e de precisão e exatidão intra e intercorridas estão apresentados na Tabela 2, abaixo. 
Tabela 2 - Valores médios de concentrações de BU em amostras de plasma obtidos no LIQ, CQB, CQM e CQA e de precisão e exatidão intra e intercorridas.

\begin{tabular}{|c|c|c|c|c|c|c|c|}
\hline \multirow[t]{2}{*}{ Nível } & \multirow{2}{*}{$\begin{array}{c}\text { Bussulfano } \\
\text { Concentração } \\
\left(n g \mathrm{~mL}^{-1}\right)\end{array}$} & \multirow[t]{2}{*}{ Lote } & \multirow{2}{*}{$\begin{array}{l}\text { Média } \pm D P \\
\left(n g \mathrm{~mL}^{-1}\right)\end{array}$} & \multicolumn{2}{|c|}{ Intracorridas } & \multicolumn{2}{|c|}{ Intercorridas } \\
\hline & & & & $\begin{array}{c}\text { Precisão } \\
(\mathrm{CV} \%)\end{array}$ & $\begin{array}{c}\text { Exatidão } \\
(\%)\end{array}$ & $\begin{array}{l}\text { Precisão } \\
(\mathrm{CV} \%)\end{array}$ & $\begin{array}{c}\text { Exatidão } \\
(\%)\end{array}$ \\
\hline & & $1(n=6)$ & $188,05 \pm 21,14$ & 11,25 & 94,02 & & \\
\hline \multirow[t]{3}{*}{ LIQ } & 200 & $2(n=6)$ & $203,76 \pm 20,84$ & 10,23 & 101,88 & 10,71 & 102,18 \\
\hline & & $3(n=6)$ & $220,18 \pm 10,37$ & 4,71 & 110,09 & & \\
\hline & & $1(n=6)$ & $261,91 \pm 3,51$ & 1,34 & 87,30 & & \\
\hline \multirow[t]{3}{*}{ CQB } & 300 & $2(n=6)$ & $276,57 \pm 3,46$ & 1,25 & 92,19 & 3,18 & 89,61 \\
\hline & & $3(n=6)$ & $267,85 \pm 9,85$ & 3,68 & 89,28 & & \\
\hline & & $1(n=6)$ & $2.049,06 \pm 32,34$ & 1,58 & 95,31 & & \\
\hline \multirow[t]{3}{*}{ CQM } & 2150 & $2(n=6)$ & $2.038,09 \pm 31,87$ & 1,56 & 94,79 & 3,40 & 97,03 \\
\hline & & $3(n=6)$ & $2.133,12 \pm 65,69$ & 3,08 & 99,21 & & \\
\hline & & $1(n=6)$ & $3.934,80 \pm 87,39$ & 2,22 & 98,37 & & \\
\hline \multirow[t]{2}{*}{ CQA } & 4000 & $2(n=6)$ & $3.896,78 \pm 86,14$ & 2,21 & 97,42 & 2,17 & 98,05 \\
\hline & & $3(n=6)$ & $3.934,27 \pm 92,16$ & 2,34 & 98,36 & & \\
\hline
\end{tabular}

Legenda: LIQ = Limite Inferior de Quantificação; CQB = Controle de Qualidade Baixo; CQM = Controle de Qualidade Médio; CQA = Controle de Qualidade Alto. Fonte: Autores.

Os resultados do teste de estabilidade de curta duração após processo de extração - $10 \mathrm{~h}$ e $6 \mathrm{~h}$ à temperatura ambiente - apresentaram-se aceitáveis, assim como os resultados do teste de estabilidade após três ciclos de congelamento e descongelamento do extraído e da solução (Tabela 3).

Tabela 3 - Estabilidade de curta duração, após ciclos de congelamento e descongelamento e de longa duração.

\begin{tabular}{cccccc}
\hline Controle & Razão $^{1}$ & Razão $^{2}$ & Razão $^{3}$ & Razão $^{4}$ & Razão $^{5}$ \\
\hline CQA & $1,05 \pm 0,07(7,5 \%)$ & $1,00 \pm 0,02(2,40 \%)$ & $1,01 \pm 0,09(9,09 \%)$ & $0,93 \pm 0,05(5,65 \%)$ & $1,05 \pm 0,06(6,31 \%)$ \\
CQB & $1,00 \pm 0,06(6,2 \%)$ & $1,10 \pm 0,03(3,18 \%)$ & $1,07 \pm 0,03(3,64 \%)$ & $0,98 \pm 0,02(2,53 \%)$ & $1,11 \pm 0,13(12,39 \%)$ \\
PI & $1,06 \pm 0,14(13,1 \%)$ & $1,01 \pm 0,04(4,13 \%)$ & $1,06 \pm 0,10(10,32 \%)$ & $0,95 \pm 0,04(4,82 \%)$ & $1,00 \pm 0,09(9,59 \%)$ \\
\hline
\end{tabular}

Legenda: $\mathrm{CQA}=$ Controle de Qualidade Alto; $\mathrm{CQB}=$ Controle de Qualidade Baixo; PI = Padrão Interno. ${ }^{1}$ (área após $10 \mathrm{~h} /$ área recémextraído) $\pm \mathrm{DP} ;{ }^{2}$ (área após $\left.6 \mathrm{~h} / a ́ r e a ~ r e c e ́ m-e x t r a i ́ d o\right) ~ \pm \mathrm{DP} ;{ }^{3}$ (área após três ciclos/área recém-extraído) $\pm \mathrm{DP} ;{ }^{4}$ (área após três ciclos/área recém-preparado) $\pm \mathrm{DP} ;{ }^{5}$ (área após 4 meses congelado/área recém-extraído) \pm DP. Valores expressos como razão de área \pm desvio padrão relativo e desvio padrão relativo (\%). Fonte: Autores.

A recuperação relativa média dos controles, obtida por intermédio da comparação dos resultados do analito

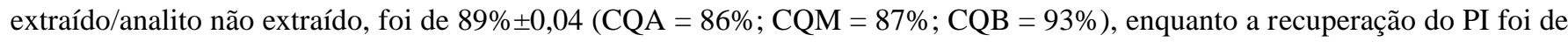
$74 \% \pm 0,47$.

A recuperação absoluta média dos controles, obtida pela comparação dos resultados do analito extraído/analito não extraído, foi de $114 \% \pm 0,03(\mathrm{CQA}=111 \%$; CQM = 118\%; CQB = 113\% $)$, ao passo que a recuperação do PI foi de $102 \% \pm 2,5$. 


\section{Discussão}

A técnica descrita foi desenvolvida a partir da otimização de técnicas clássicas, utilizadas em métodos cromatográficos previamente descritos para análise de bussulfano em HPLC (Chow et al., 1997; Heggie et al., 1997; Bleyzac et al., 2000; Tran et al., 2000). O desenvolvimento desta técnica envolve a eliminação dos interferentes endógenos a partir da precipitação de proteínas com acetonitrila, a qual demonstrou maior eficiência que o metanol, pois produz sobrenadante mais límpido e não interfere nos picos cromatográficos, além do que, o metanol pode levar à hidrólise dos compostos derivatizados (Chow et al., 1997; Bleyzac et al., 2000).

A técnica demonstrou ter boa seletividade frente ao desafio de identificar e de quantificar o bussulfano, quando desafiado por amostras de plasma normais, lipêmicas e hemolisadas, além de amostras adicionadas de 14 diferentes fármacos, comumente administrados a pacientes internados para a realização do transplante de medula óssea. Outrossim, a técnica desenvolvida atende aos propósitos de controle terapêutico para o bussulfano, fato descrito também por autores como Chow et al. (1997), Heggie et al. (1997), Bleyzac et al. (2000) e Quernin et al. (1999). A recuperação média obtida (89\% $\pm 0,04)$ foi superior à descrita por outros autores, de 70 $\pm 5 \%$ e $78 \%$ (Quernin et al., 1997), ressaltando-se que a utilização do padrão interno corrige eventuais perdas e oscilações no processo de extração do analito.

$\mathrm{O}$ valor de sensibilidade obtido foi menor do que o descrito por Bleysac et al (2000) (25 ng mL-1), porém maior do que o registrado por Heggie et al. (1997) $\left(60 \mathrm{ng} \mathrm{mL}^{-1}\right)$. Entretanto, isso não pode ser considerado como uma desvantagem do método, pois pelo descrito na literatura acerca da farmacocinética do bussulfano, a sensibilidade de $200 \mathrm{ng} \mathrm{mL}^{-1}$ é suficiente para detectar as concentrações terapêuticas (600 a $900 \mathrm{ng} \mathrm{mL}^{-1}$ ), subterapêuticas e supraterapêuticas deste fármaco (Salman et al., 2019; Philippe et al., 2019).

Os valores de exatidão e precisão encontrados na literatura (Chow et al., 1997; Heggie et al., 1997; Peris et al., 1999; Bleyzac et al., 2000; Hara et al., 2000) assemelham-se aos dados obtidos no presente estudo (Tabela 2) e estão abaixo dos limites estabelecidos pela RE n ${ }^{\circ}$ 899/2003, da ANVISA (Brasil, 2003), para métodos bioanalíticos (precisão até 20\% para o LIQ e $15 \%$ para os demais pontos da curva de calibração, e exatidão de 80 a 120\%). Com referência à mesma Resolução acrescenta-se, ainda, que o processo de validação preencheu todos os critérios de aceitação da curva de calibração, incluindo valores de precisão e de exatidão para cada ponto e o coeficiente de correlação linear, que deve ser igual ou superior a 0,98.

Balasubramanian et al. (2001) demonstraram que o bussulfano é estável em plasma por mais de 3 meses a $-20^{\circ} \mathrm{C}$ e que as amostras de sangue para análise deste composto podem ser centrifugadas até $3 \mathrm{~h}$ após a coleta. Adicionalmente, o bussulfano é estável em sangue total por $24 \mathrm{~h}$ a $4^{\circ} \mathrm{C}$ e em plasma por até 2 anos a $-80{ }^{\circ} \mathrm{C}$ (Balasubramanian et al., 2001). Acrescenta-se que, como apresentado na Tabela 3, os valores obtidos para estabilidade, incluindo de longa duração, apresentam desvios sempre inferiores a 15\% (Brasil, 2003), demonstrando que as eventuais reduções de concentração, tanto do analito bussulfano, quanto do padrão interno, são completamente aceitáveis. Por outro lado, o tempo total de corrida analítica para um paciente (quatro amostras, em duplicata), incluindo os procedimentos de extração e derivatização, bem como a injeção de controles (CQB, CQM e CQA) e de branco, é de cerca de $10 \mathrm{~h}$. Esse tempo é suficiente para fazer intervenção na posologia do bussulfano durante os 4 dias de tratamento. Por outro lado, a realização do chamado pré-teste (uma dose de bussulfano antes do início do tratamento definitivo), faculta a obtenção de parâmetros farmacocinéticos que podem ser utilizados para prever a dose mais adequada para o tratamento.

A técnica descrita no trabalho apresenta a vantagem de necessitar de curto tempo de análise $(17 \mathrm{~min})$, com cromatograma livre de interferentes; utiliza apenas $500 \mu \mathrm{L}$ de plasma e apresenta baixo custo, pois a extração é líquida-líquida e a preparação da amostra conta com apenas duas etapas, uma de precipitação de proteínas e outra de extração com acetato de etila. 
Essa técnica pode ser aplicada a estudos farmacocinéticos e de monitoração terapêutica em pacientes submetidos a condicionamento com este fármaco para transplante de TCTH, em tempo hábil para que mudanças de posologia, se necessárias, possam ser implementadas ainda durante o tratamento, conforme demonstrado por Effting et al. (2015).

\section{Conclusão}

A validação da técnica analítica, em HPLC-PDA, para dosagem plasmática do bussulfano em amostras de pacientes que se subeteram à ablação de medula óssea, apresentou parâmetros de validação dentro dos limites estabelecidos pela legislação brasileira vigente, sendo adequada para a utilização pleiteada, que envolve estudos de monitorização terapêutica e de farmacocinética clínica do bussulfano.

\section{Agradecimentos}

Os autores agradecem à Fundação de Amparo à Pesquisa do Estado de Goiás (FAPEG) e ao Conselho Nacional de Ciência e Tecnologia (CNPq) pelo apoio financeiro.

\section{Referências}

Balasubramanian, P., Srivastava, A., Chandy, M. (2001). Stability of busulfan in frozen plasma and whole blood samples. Clinical Chemistry, 47(4):766-8.

Bleyzac, N, Barou P, Aulagner G. (2000). Rapid and sensitive high performance liquid chromatographic method for busulfan assay in plasma. Journal of Chromatography B Biomedical Sciences and Applications, 742(2):427-32.

Bornhäuser, M. et al. (2003). Conditioning with fludarabine and targeted busulfan for transplantation of allogeneic hematopoietic stem cells. Blood., 102(3):820-6.

Brasil. Ministério da Saúde. (2003). Agência Nacional de Vigilância Sanitária. Resolução - RE nº 899, de 29 de maio de 2003. Diário Oficial da União, Brasília (DF).

Bullock, J. M., Smith, P. F., Booker, B. M., Loughner, J., Capozzi, D., Mccarthy, Junior, P. L., Shaw, L. M. (2006). Development of a pharmacokinetic and Bayesian optimal sampling model for individualization of oral busulfan in hematopoietic stem cell transplantation. Therapeutic Drug Monitoring, 28(1):62-6.

Chow, D. S. L., Bhagwatwar, H. P., Phadungpojna, S., Andersson, B. S. (1997). Stability-indicating high-performance liquid chromatographic assay of busulfan in aqueous and plasma samples. Journal of Chromatography B Biomedical Sciences and Applications., 704(1-2):277-88.

Ciurea, S. O., Andersson, B. S. (2009). Busulfan in hematopoietic stem cell transplantation. Biologic Blood Marrow Transplantation, $15(5): 523-36$.

Corbacioglu, S., Jabbour, E. J., Mohty, M. (2019) Risk Factors for Development of and Progression of Hepatic Veno-Occlusive Disease/Sinusoidal Obstruction Syndrome. Biological Blood Marrow Transplantation. 2019 Jul;25(7):1271-1280.

Effting, C., Arantes, A. M., Labre, L.V. Q., Carneiro, W. J., Oliveira-Neto, J. R., Bariani, C., Rodrigues, C. R., Rodrigues, A. R., Cunha, L. C. (2015). Individualizing oral busulfan dose after using a test dose in patients undergoing hematopoietic stem cell transplantation: pharmacokinetic characterization. Therapeutic Drug Monitoring, 37 (1), 66-70.

Feng, X., Wu, Y., Zhang, J., Li, J., Zhu, G., Fan, D., Yang, C., Zhao, L. (2020) Busulfan systemic exposure and its relationship with efficacy and safety in hematopoietic stem cell transplantation in children: a meta-analysis. BMC Pediatrics. Apr 20;20(1):176.

Hara, S., Tsuchie, M., Tsujioka, R., Kimura. M., Fuji, M., Kuroda, T., Ono, N. (2000). High-performance liquid chromatographic quantification of busulfan in human serum after fluorescence derivatization by 2-naphthalenethiol. Analytical Sciences, 16(3):287-91.

Hassan, M. et al. (2000). The effect of busulphan on the pharmacokinetics of cyclophosphamide and its 4-hydroxy metabolite: time interval influence on therapeutic efficacy and therapy-related toxicity. Bone Marrow Transplantation, 25(9):915-24.

Heggie, J. R., Wu, M., Burns, R. B., Ng, C. S., Fung, H. C., Knight, G., Barnett, M. J., Spinelli, J. J., Embree, L. (1997). Validation of a high-performance liquid chromatographic assay method for pharmacokinetic evaluation of busulfan. Journal of Chromatography B Biomedical Sciences and Applications, 692(2):437-44.

ICH Harmonised Tripartite Guideline. (2005). International Conference on Harmonization of Technical Requirements for Registration of Pharmaceuticals for Human Use, Geneva, Switzerland.

Lawson, R., Staatz, C. E., Fraser, C. J., Hennig, S. (2021) Review of the Pharmacokinetics and Pharmacodynamics of Intravenous Busulfan in Paediatric Patients. Clinical Pharmacokinetics. Jan; 60(1):17-51.

Nath, C. E., Shaw, P. J. (2007). Busulfan in blood and marrow transplantation: dose, route, frequency, and role of therapeutic drug monitoring. Current Clinical Pharmacology, 2(1):75-91. 
Peris JE, Latorre JA, Castel V, Verdeguer A, Esteve S, Torres-Molina F. (1999). Determination of busulfan in human plasma using high-performance liquid chromatography with pre-column derivatization and flurescence detection. Journal of Chromatography B: Analytical Technologies in the Biomedical and Life Sciences, 730(1):33-40.

Philippe, M., Neely, M., Rushing, T., Bertrand, Y., Bleyzac, N., Goutelle, S. (2019) Maximal concentration of intravenous busulfan as a determinant of venoocclusive disease: a pharmacokinetic-pharmacodynamic analysis in 293 hematopoietic stem cell transplanted children. Bone Marrow Transplantation. Mar;54(3):448-457.

Quernin, M. H., Poonkuzhali, B., Médard, Y., Dennison, D., Srivastava, A., Krishnamoorthy, R., Chandy, M., Jacqz-Aigrain, E. (1999). High-performance liquid chromatographic method for quantification of busulfan in plasma after derivatization by tetrafluorotiophenol. Journal of Chromatography B: Analytical Technologies in the Biomedical and Life Sciences, 721(1):147-52.

Salman, B., Al-Khabori, M., Al-Huneini, M., Al-Rawas, A., Dennison, D., Al-Za'abi, M. (2020) Busulfan clearance does not predict the development of hepatic veno-occlusive disease in patients undergoing hematopoietic stem cell transplantation. International Journal of Hematology, Aug;112(2):210-216.

Slattery, J. T. et al. (1997). Marrow transplantation for chronic myeloid leukemia: the influence of plasma busulfan levels on the outcome of transplantation. Blood., 89(8):3055-60.

Slattery, J. T., Risler, L. J. (1998). Therapeutic monitoring of busulfan in hematopoietic stem cell transplantation. Therapeutic Drug Monitoring, 20(5):543-49.

Tabak, A., Hoffer, E., Rowe, J. M., Krivoy, N. (2001). Monitoring of busulfan area under the curve: estimation by a single measurement. Therapeutic Drug Monitoring, 23(5):526-8.

Takamatsu, Y., Ogata, K., Yamauchi, K., Hara, S., Kamimura, T., Hayashi, S., Suzumiya, J., Tamura, K. (2005). An evaluation of busulfan pharmacokinetics in patients undergoing hematopoietic stem cell transplantation. Japanese Journal of Clinical Oncology, 35(7):400-3.

Tran, H. T., Madden, T., Petropoulos, D., Worth, L. L., Felix, E. A., Sprigg-Saenz, H. A., Choroszy, M., Danielson, M., Przepiorka, D., Chan, K. W. (2000). Individualizing high-dose oral busulfan: prospective dose adjustment in a pediatric population undergoing allogeneic stem cell transplantation for advanced hematologic malignancies. Bone Marrow Transplantation, 26(5):463-70. 